\title{
EQUIPMENT FOR DISTRIBUTING GRANULAR INSECTICIDES
}

\author{
M. W. CRoss \\ Massey University of Manawatu, Palmerston North
}

\section{Summary}

The efficiency of the equipment available for the distribution of granular insecticides at low rates per acre is discussed, and queries are raised regarding the standard of accuracy required in the field so that calibration tests will have some real significance.

THE PRACTICAL INTERPRETATION of the results obtained during static and field tests of the evenness of distribution from fertilizer distributors has always been a major obstacle to the evolution of a realistic "standard" method of testing. N.I.A.E. Report No. 354, "A Series Test of Fertilizer Broadcasters, 1963-64, Part 1", states: "At present only an approximate assessment can be given of the degree of variation in application rate which can be tolerated by crops. It is certain that a perfectly even distribution is unnecessary; however, it is considered that a variation of $\pm 33 \%$ of the average application rate is the maximum variation that is acceptable, particularly under test conditions, which were ideal".

Is this degree of accuracy acceptable for the distribution of granular insecticides - whether discharged along rows of crops or broadcast? If not, then what is an acceptable distribution pattern?

Since the gazetting of the Agriciltural Chemicals (Insecticides) Regulations 1964, emphasis has been placed on the need for accurate and uniform distribution, especially for the control of grass-grub (Costelytra zealandica) and porina caterpillar (Wiseana spp.) when the granules are broadcast. If absolute accuracy is demanded, then current equipment is not acceptable. But, if the permissible variation suggested for fertilizer distributors is within acceptable limits, then it may be satisfactory.

A recently extracted sample of commercial DDT prills was found to contain approximately 2,400 prills per gram. If this material is to be applied uniformly at the rate of $10 \mathrm{lb}$ per acre, approximately 1.75 prills would need to be deposited on each sqiaare inch of ground surface. For a perfect distribution, the prills would need to be placed in a definite pattern so that 7 prills were equally spaced over each 4 sq. in., provided always that each prill contained exactly the same amount of active insecticide.

In this sample, the variation in prill size was relatively quite large, the largest being 6 to 8 times the size of the smallest, a variation greater than that found in a sample of turnip, rape or choumoellier seed. The seeds, however, all have the ability to become established plants - has each prill the same insecticidal effect?

The main question at issue in this discussion is the degree of precision required from distributing appliances. 


\section{MECHANISMS AVAILABLE}

\section{Row APPLICATORS}

The rate of application by the spinning disc applicator which is being used for spreading prills is controlled primarily by gravity flow through a restricted outlet. If this is satisfactory, then the same control should silffice for row applicators when using freerunning granules. If this is not the case, then, logically, the current disc applicator is not acceptable. Working conditions when planting potatoes are much more conducive to a uniform gravity flow than conditions when broadcasting over pasture where higher forward speeds and rougher ground conditions are involved. When

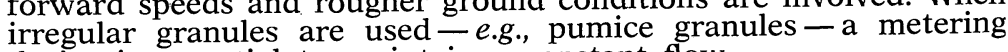
device is essential to maintain a constant flow.

Two metering mechanisms which have been in use for row applications were tested for uniformity of distribution along the row, by weighing the material collected in 3 in. divisions of forward movement. With disulfoton in pumice granules, the Horstine Farmery "Microband" applicator showed a maximum variation of $+21 \%$ $-31 \%$ from the mean rate. If 6 in. divisions had been the criterion, the maximum variations would then have been $+10 \%-21 \%$.

This compares favourably with the N.I.A.E. Test Report No. 328, in which a variation, for 3 in. divisions, of $\pm 30 \%$ from the mean rate was considered acceptable since the peaks occurred at short intervals and were never more than $4 \frac{1}{2} \mathrm{in}$. away from any plants in the row.

For 3 in. divisions, the A. H. Dale prototype applicator showed a maximum variation of $+17 \%-32 \%$ from the mean rate. Increased to a 6 in. step, this becomes $+10 \%-18 \%$. Production models should have a still better performance with the elimination of the minor faults in the prototype.

It should be remembered, however, that the insecticide granules in row application are placed in a narrow band. Even if they were spread in a band 9 to 12 in. wide, there would still be some 10 granules per sq. in. and the slight variations of $20 \%$ of the mean ( 3 in. divisions) should have little real importance.

\section{BroAdCAST APPLICATORS}

Equipment for broadcast application has to work under much more difficult conditions - thus, logically, it should be much more precise. Unfortunately, the reverse appears to be the case. All too often little real care is taken to ensure there is a uniform spread. Provided the granules can be seen spreading into the air behind a "throwing" distributor, it is too often taken for granted that the spread is sufficiently accurate.

Two types of distributors are currently employed, the spinning disc - e.g. Tartan Speed Spreader - and the reciprocating nozzle e.g., Vicon Varispreader. Static tests of these indicate a transverse distribution (for a $14 \mathrm{ft}$ spreading width) with maximum variations from the mean rate of $+32 \%-24 \%$ (Tartan), and $+21 \%-37 \%$ (Vicon) when measured in 5 in. divisions (D. Rastrick, pers. comm.). However, the longitudinal distribution pattern was not investigated.

The transverse evenness of distribution is only one aspect of the machine's performance, and may be counter-balanced by other faults or virtues.

Longitudinal distribution is obviously as important and variations in it usually reflect the reactions of the feed mechanism to changes in the degree of slope, bumping or vibration during work, or to 
changes in the physical properties of the granules in the hopper. Additionally, the effect of wind and inaccurate driving may be very pronounced.

\section{SOME OF THE FACTORS AFFECTING THE DISTRIBUTION OF "THROWING" DISTRIBUTORS}

\section{Feed Mechanisms}

The quantity of material delivered to the spreading mechanism should be related to the distance traversed. A metering device driven by a land wheel is therefore most desirable. If a gravity feed through a restricted outlet is relied upon, the rate can be expected to vary with variation in the amount of material in the hopper, the influence of bumps and vibration, and slight changes in moisture content. An assisted gravity feed, such as an agitator feeding through a restricted outlet, reduces the severity of these variations but both types are completely dependent, for uniformity of longitudinal distribution, on a constant forward speed being maintained at all times. In the field, a local variation of i m.p.h. - and this is very common can cause up to a $30 \%$ or more change in the delivery rate. The faster the forward speed, the more difficult it becomes to maintain a uniform speed.

\section{SPREADING MEchanisms}

When material is thrown from a spreading mechanism, it is subject to many influences before it reaches the ground. Transverse distribution is not uniform for a spinning disc, being heavier on the side toward which the disc is rotating. For the reciprocating nozzle distributor, provided particle sizes do not vary widely, a reasonably uniform swath may be expected under static conditions, with a sharp drop-off at the edges.

\section{Overlap}

When using full width distributors, little or no overlapping of the edges of siccessive runs is required in order to obtain the best possible evenness of transverse distribution. When "throwing" machines are used, it is necessary to overlap the edges of successive runs. If a field is sown round and round and uniform bout widths maintained, material from the heavy side of a spinning-disc swath will fall on to the light side of the swath of the previous bout. This will give a more uniform overall distribution than if the field is sown back and forth - unless the bout widths are varied for each run (Hepherd and Pascal, 1958)

If a bout width of 28 to $30 \mathrm{ft}$ is selected, it becomes virtually impossible for a driver to maintain a uniform spacing inless driving to accurately-placed markers for each run. It would appear desirable to limit the bout width to 14 to $15 \mathrm{ft}$ with spinning-disc distributors, so that each portion of the field is covered twice at half the rate. For the reciprocating nozzle distributor, the sowing pattern falls away sharply and reasonably uniformly at the edges of the $14 \mathrm{ft}$ swath - thus maintenance of correct overlap is easier whether travelling round and round or back and forth across the field.

\section{Segregation of Particles}

The further particles are thrown, the greater the degree of segregation of the various sizes (Crowther, 1958). This is influenced by 
disc-speed (peripheral speed) and at 800 r.p.m. ( $52 \mathrm{ft} / \mathrm{sec}$ ) can be expected to be large, in view of the variation in the sizes of particles found in the sample mentioned earlier. The ideal overlap would be such that the small particles from one run meet the small particles from the previous run.

\section{Effect of Wind}

The effect of a cross-wind on the transverse distribution pattern from a spinning-disc can be very marked, more especially if bout widths are of the order of $30 \mathrm{ft}$. Under field conditions, where wind strength and direction are likely to vary from place to place in the field, it can be expected to cause quite large areas to receive little or no application, and the narrower bout wiaths are thus recommended. Even then, the spread from both types of distributors will be affected to some extent, unless the feed to the spreading mechanism is from a ground-wheel drive.

\section{Effect of Slope}

With the reciprocating nozzle distributor, a reasonably symmetrical pattern may be expected whether on level ground or travelling up, down or across a slope, provided the distributing mechanism remains parallel with the ground surface. For the spinning disc, however, changes from the horizontal alter the point at which the particles fall on to the disc, thus altering the radial distribution pattern.

\section{Effect of Speed Changes}

With both these distributors, it is essential that the spreading mechanism be kept revolving or oscillating at a constant rate, otherwise the effective width of spread is altered and the efficiency of longitudinal distribution lowered. The ideal woild be an independent constant speed drive, whether it be by electric, hydraulic or internal combustion motor. If a power take-off drive is employed, a tachometer or speedometer and an efficient governor on the engine are essential to maintain a constant engine speed, and it is necessary to remain in the same gear all the time.

\section{CONCLUSIONS}

The equipment mentioned previously for row crop application of granular insecticides appears to have an acceptable performance.

Under field conditions, broadcast applicators are influenced by so many variables that the poor results reported following their use for spreading granular insecticide for grass-grub control may, in many cases, be attributable largely to the lack of a precisely uniform coverage. Other types of applicators involving the use of fixed width distributors with positive feed mechanisms may be warranted at the least, for treatment of smooth-surface high-production pastures for grass-grub control.

The uniformity of the transverse and longitudinal distribution of material from distributors is commonly checked by weighing the amounts collected in segments of the transverse or longitudinal spread. When considering the efficiency of distribution of granular insecticides by broadcast applicators, the number and precise position of the granules is of more import than their weight. Thus a more accurate and meaningful method of calibration would be an indication of the number of granules deposited in small subdivi- 
sions of the spread - the size of the subdivisions depending upon the diffusion characteristics of the insecticide.

No condemnation of current broadcasting equipment is implied in this discussion; the aim has been to indicate its shortcomings for this specialized work. Many of the inherent inaccuracies are exaggerated in the field, sometimes by carelessness on the part of the operator, at other times by circumstances beyond his control. Just how often all the requirements for an accurate spread by these types of distributors are fulfilled in practice can best be left to the imagination. It is certain that better overall results with this equipment could be obtained if the work were done always by skilful and conscientious operators. As this is not the case, would not the availability for hire of fixed width equipment with improved inherent distribilting qualities be warranted?

Finally, is it possible to specify the standard of accuracy required of granule distributors for both broadcast and row applications?

\section{REFERENCES}

Crowther, A. J., 1958: J. agric. Eng. Res., 3 (3): 288.

Hepherd, R. Q.; Pascal, A. J., 1958: J. agric. Eng. Res., 3 (2): 95. 